\title{
Equivalência semântica, conceitual e de itens do Observable Indicators of Nursing Home Care Quality Instrument
}

\author{
Semantic and conceptual equivalence of the observable indicators \\ of Nursing Home Care Quality Instrument
}

Wagner Ivan Fonseca de Oliveira ${ }^{1}$
Pedro Jesus Saturno Hernández ${ }^{2}$
Kelienny de Meneses Sousa $^{3}$
Grasiela Piuvezam $^{3}$
Zenewton André da Silva Gama $^{3}$
${ }^{1}$ Instituto Federal de Educação Ciência e Tecnologia do Rio Grande do Norte. RN-288 s/n, Nova Caicó. 59300-000 Caicó RN Brasil. wagner.fonseca@ifrn.edu.br. ${ }^{2}$ Unidad de Medicina Preventiva y Salud Pública, Universidad de Murcia. Murcia Espanha.

${ }^{3}$ Departamento de Saúde Coletiva, Universidade Federal do Rio Grande do Norte. Natal RN Brasil.

\begin{abstract}
Long-term care facilities (LTCFs) for the elderly are an important health care alternative in the world, though Brazil does not yet have a valid instrument to monitor the quality of these institutions. This study sought to describe the initial stages of the cross-cultural adaptation of the Observable Indicators of Nursing Home Care Quality (OIQ) instrument used to assess the quality of care in LTCFs. Conceptual equivalence was conducted to assess the relevance and feasibility of the OIQ within the Brazilian context, using the Content Validity Index (CVI). The operational, idiomatic and semantic equivalence was then performed. This item consisted of 5 phases: (1) two translations; (2) the respective back translations; (3) formal appraisal; (4) review; and (5) application of the pre-test in three LTCFs. Significant changes were made to ensure the validity of the OIQ. The CVI instrument for the Brazilian contextwas $94.3 \%$ (viability) and $95.3 \%$ (relevance). The OIQ proved to be easy to understand and apply in the pre-test. Cross-cultural adaptation of the OIQ contributes to assessing and improving quality in Brazilian LTCFs, though the findings should be complemented by a psychometric evaluation of the instrument.

Key words Cross-cultural comparison, Longterm care facilities, Quality indicators, Quality of healthcare
\end{abstract}

Resumo As Instituições de Longa Permanência para Idosos (ILPI) são uma importante alternativa de cuidado no mundo, porém o Brasil ainda não dispõe de instrumento válido para monitorar a qualidade dessas instituições. Portanto, o objetivo do presente artigo é descrever as etapas iniciais da adaptação transcultural do Observable Indicators of Nursing Home Care Quality Instrument (IOQ) usado para avaliar a qualidade do cuidado nas ILPI. Realizou-se a equivalência conceitual e de itens para avaliar a pertinência e a viabilidade do IOQ à realidade nacional através do Indice de Validade do Conteúdo (IVC). Em seguida, cumpriu-se a equivalência operacional, a idiomática e a semântica. Esta última tem 5 fases: (1) duas traduções e (2) duas respectivas retraduções; (3) apreciação formal; (4) revisão; e (5) aplicação do pré-teste em três ILPI. Modificações importantes foram realizadas para garantir a validade do IOQ. O IVC do instrumento referentes ao contexto brasileiro foi de $94,3 \%$ (viabilidade) $e$ 95,3\% (relevância). O IOQ mostrou-se compreensivel e de fácil aplicação no pré-teste. A adaptação transcultural do IOQ contribui para avaliação e melhoria da qualidade nas ILPI brasileiras, mas os resultados devem ser complementados mediante avaliação psicométrica.

Palavras-chave Comparação transcultural, Instituição de longa permanência para idosos, Indicadores de qualidade, Qualidade da assistência à saúde 


\section{Introdução}

O envelhecimento populacional é um dos mais relevantes desafios da saúde pública atual. Chegar à velhice é uma realidade populacional mesmo nos países mais pobres. No Brasil, esse fenômeno vem ocorrendo associado a um contexto de envelhecimento da própria população idosa, devido a um crescimento mais acentuado do contingente populacional de 80 anos ou mais ${ }^{1}$; de mudanças nos arranjos familiares e no papel social da mulher pela inserção notável no mercado de trabalho; e de níveis de fecundidade reduzidos $^{2}$. Esses processos resultam no aumento da população daqueles que demandam cuidados prolongados, acompanhado de uma redução da oferta de atenção por familiares.

Dentre as alternativas ao cuidado do idoso, as instituições asilares são as mais antigas.Recentemente foram renomeadas como Instituições de Longa Permanência para Idosos (ILPI). Trata-se de uma adaptação do termo utilizado pela Organização Mundial de Saúde (Long-Term Care Institution $)^{3}$. Essa mudança visou uma conotação híbrida da função dos "lares de idosos" por englobar, além da função social, serviços de atenção à saúde. Mesmo enraizadas por preconceitos culturais acentuados e por uma frequente baixa qualidade dos serviços ${ }^{4,5}$, o número de ILPI no Brasil vem crescendo consideravelmente ${ }^{6}$, sendo de extrema importância conhecer melhor e regular a qualidade deste segmento de institucionalização.

Historicamente, a melhoria da qualidade dos serviços nas ILPI vem sendo foco de importantes iniciativas de políticas públicas internacionais, debates de consumidores e relatórios profissionais ${ }^{7,8}$. Define-se qualidade como um serviço ou produto "adequado à utilização pretendida" Instituto de Medicina (IOM), dos Estados Unidos da América (EUA), descreve a qualidade na assistência à saúde como o grau em que os serviços aumentam a probabilidade de obter os resultados desejados com o nível de conhecimento científico atual, abrangendo seis dimensões para caracterizar o desempenho em saúde: segurança, eficiência, efetividade, atenção centrada no paciente, equidade e acesso ${ }^{8}$. Na esfera das ILPI, Rantz et al. ${ }^{10}$ propuseram um modelo para avaliar a qualidade do cuidado a partir de um conceito multidimensional envolvendo dimensões alusivas às características do cuidado, meio ambiente, envolvimento familiar, comunicação com foco central nos residentes, familiares e comunidade na qual está inserida a instituição, prio- rizando também a equipe de trabalho por estar diretamente vinculada à prestação de cuidados.

A dimensão da "atenção centrada no paciente", mais recentemente chamada de "atenção centrada na pessoa", por abranger não somente aspectos clínicos, mas também questões sociais e culturais, é utilizada como referência para avaliar a qualidade nas ILPI e reconhecida mundialmente por importantes instituições ${ }^{8,11,12}$. De acordo com a Aliança Internacional das Organizações dos Pacientes (AIOP), o elemento mais comumnas definições da atenção centrada na pessoa é o respeito pelas necessidades, desejos, referências e valores dos pacientes ${ }^{13}$, semelhante à definição do IOM, que a considera um cuidado respeitável e responsivo às preferências individuais dos pacientes, necessidades e desejos, garantindo que os valores individuais dos usuários possam orientar todas as decisões clínicas ${ }^{8}$. Porém, o empoderamento das pessoas e familiares quanto às tomadas de decisões, podem frustrar os prestadores de serviços gerando conflitos e dificuldade para sua efetiva implantação ${ }^{14}$.

Recentes pesquisas têm apresentado diversos benefícios relacionados a esta dimensão, amplamente caracterizados pelo aumento da qualidade e da segurança nos cuidados à saúde, pela diminuição dos custos ${ }^{13,15-17}$ e nas condições crônicas: aumento nos padrões de funcionalidade e qualidade de vida ${ }^{14,17,18}$. Ainda que outras definições as defendam como uma dimensão em seu próprio direito e primária de qualidade própria ${ }^{19}$, também é verdade que a maioria dos pesquisadores que a estudaram sistematicamente evidenciaram resultados positivos em relação aos clássicos resultados de saúde ${ }^{20}$.

Embora estudos qualitativosrecentestenham exploradoa experiênciada vida cotidianados residentes das ILPI em cuidados de longa permanência ${ }^{21}$, incluindo a que contribui para a adaptação à vida na instituição ${ }^{22}$ e para a qualidade de vida $^{23}$, não abrangem o entendimento dos usuários quanto à qualidade do cuidado.

Nesse contexto, Rantz et al..$^{10}$ elaboraram um modelo conceitual relacionando a qualidade do cuidado nas ILPI a partir da atenção centrada na pessoa. Desenvolveu-se através de estudos qualitativos abrangendo não apenas as percepções da qualidade por especialistas e prestadores de cuidados, mas também pelos usuários e familiares. A partir de então, constitui-se o instrumento dos Indicadores Observáveis da Qualidade do Cuidado nas ILPI (IOQ), de caráter multidimensional após estudos de campo em 530 ILPI abrangendo uma pequena amostra internacional e institui- 
ções de três estados norte-americanos, durante visitas de 30 a 40 minutos $^{24,25}$.

O IOQ encontra-se em sua décima versão ${ }^{25}$ sendo composto por 30 itens alusivos a sete dimensões da qualidade do cuidado: (1) comunicação interpessoal; (2) prestação de cuidados; (3) gestão da aparência dos idosos; (4) odores; (5) utilização dos ambientes básicos; (6) acesso aos ambientes das ILPI; e (7) ambiente familiar, priorizando indicadores de processo através de cinco dimensões $(1 ; 2 ; 3 ; 6 ; 7)$ associados a 23 itens e dados de estrutura a partir de duas dimensões $(4 ; 5)$ constituídas por 7 itens. Para cada um dos itens são atribuídos pontuações de 1 a 5 em ordem crescente de qualidade relacionada a aspectos diretamente observáveis nas instituições, apresentando variação de um mínimo de 30 (má qualidade) a um máximo de 150 pontos (boa qualidade) por instituição.

Apesar da importância das Instituições de Longa Permanência para Idosos como alternativa de cuidados em todo mundo, no Brasil ainda não dispõe de instrumento válido e confiável para monitorar a qualidade nesse contexto. Encontramos apenas dois instrumentos de avaliação da qualidade nessas instituições: (1) fundamentado na Resolução da Diretoria Colegiada (RDC) No. 283/2005, da Agência Nacional de Vigilância Sanitária ${ }^{26}$, que define condições mínimas de funcionamento das ILPI, e (2) uma ferramenta avaliativa ${ }^{27}$ baseada em regulamentações do conselho estadual do Rio Grande do Sul de enfermagem e da RDC 283/2005. Além da ausência de validação, ambas se limitam por uma considerável especificidade, pois contemplam, principalmente, indicadores de estrutura ${ }^{26}$ ou pelo direcionamento exclusivo aos enfermeiros em um contexto complexo e multiprofissional das ILPI $^{27}$. Evidencia-se assim, a necessidade de adaptar o IOQ à cultura brasileira, para favorecer a produção de informações que guiem a tomada de decisão por diferentes entes sociais: gestores, reguladores, prestadores de serviços, profissionais de saúde e potenciais consumidores. A partir de um instrumento que seja válido e confiável.

Esse estudo tem por objetivo descrever as primeiras etapas da adaptação transcultural do IOQ para a cultura brasileira apresentando uma versão síntese para posterior avaliação de suas propriedades psicométricas. Espera-se contribuir para o monitoramento da qualidade do cuidado nas ILPI e subsidiar a tomada de decisão de diferentes entes sociais, desde os potenciais consumidores aos administradores das instituições.

\section{Metodologia}

\section{Desenho do Estudo}

Trata-se de um estudo de adaptação transcultural de instrumento de medida. Apesar de haver apreciações amplas sobre o processo de adaptação transcultural, ainda não há um consenso quanto às estratégias de execução. Este estudo foi baseado em análises metodológicas de clássicos autores a respeito da adaptação transcultural ${ }^{28-31}$. Constituiu-se a partir das etapas (1) avaliação da equivalência conceitual e de itens; (2) equivalência idiomática, semântica (tradução, retradução, apreciação formal, análise crítica por um comitê de especialista e pré-teste) e a equivalência operacional.

\section{Equivalência conceitual e de itens}

Após um embasamento a partir de uma revisão de literatura do instrumento original, analisou-se a validade de face e conteúdo mediante um comitê de especialistas multidisciplinar, seguindo recomendações metodológicas ${ }^{32,33}$. Este foi constituído por 10 avaliadores associados diretamente ao objeto de estudo e representando diferentes entes sociais: pesquisadores da área da Gerontologia, administradores, profissionais de saúde e potenciais consumidores dos serviços em saúde ofertados pelas ILPI (um especialista em gestão da qualidade em serviços de saúde e uma pesquisadora em epidemiologia; dois fisioterapeutas, uma nutricionista; uma idosa; um diretor -presidente de ILPI; uma coordenadora técnica; e uma responsável pela capacitação de recursos humanos de uma ILPI, além de uma educadora física, destes, dois são doutores e dois mestres).

Utilizou-se a Técnica de Grupo Nominal adaptada $^{34}$ para pontuar cada item em relação às relevâncias alusivas às dimensões respectivas do IOQ e à viabilidade ao contexto brasileiro mediante uma escala tipo Likert de 1 a $4 \mathrm{em}$ ordem crescente de conformidade, em que: 1 (irrelevante ou inviável); 2 (pouco relevante ou pouco viável); 3 (relevante ou viável); e 4 (muito relevante ou muito viável). Calculou-se o Índice de Validade de Conteúdo por itens (IVC-I) mediante a soma de concordância dos itens que foram pontuados pelos avaliadores. Os itens que receberam pontuação " 1 " ou " 2 " foram considerados reprovados, enquanto que os " 3 " ou " 4 " corresponderam às aprovações. Dessa forma, o IVC tem sido definido como "a proporção de 
itens que receberam uma pontuação de 3 ou 4 pelos avaliadores" 35 .

Não havendo consenso sobre a validade de um dos itens na primeira votação, realizou-se uma discussão com os presentes no intuito de aceitar, modificar, substituir ou retirar os itens com IVC-I (índice de validade de conteúdo por item) menores que 0,80 ou $80 \%{ }^{36}$. Posteriormente, houve uma segunda votação para o consenso final, a fim de se chegar a uma conformidade entre os pares.

Para avaliar o índice de validade de conteúdo do questionário completo (IVC-Q) utilizou-se a média aritmética a partir da contagem dos IVC -I. O parâmetro de aprovação foi de no mínimo $90 \%{ }^{36}$.

\section{Equivalência semântica, idiomática e operacional}

A equivalência semântica, idiomática e operacional foi desenvolvida a partir das seguintes fases: tradução, retradução, apreciação formal, revisão por um segundo comitê de especialistas e o pré-teste do instrumento (Figura 1).

As questões do IOQ foram, inicialmente, traduzidas de forma independente para a língua portuguesa por duas tradutoras brasileiras fluentes em inglês. Devido ao fato do Brasil ser um país com raízes culturais heterogêneas, fez-se necessária a inclusão de tradutoras com formações culturais diversificadas ${ }^{31}$. Uma profissional com graduação em letras e mestranda da área de Linguística Aplicada foi a responsável pela tradução 1 (T1) enquanto outra, natural do Rio de Janeiro, com graduação em fisioterapia,fez a tradução 2 (T2).

Posteriormente, as traduções (T1 e T2) foram retraduzidas para o inglês (R1 e R2) por outros dois tradutores naturais dos Estados Unidos e fluentes no português brasileiro. Estas fases (R1 e R2), assim como as anteriores (T1 e T2), ocorreram de forma independente e cega em relação aos perfis profissionais que atuaram previamente.

\section{Apreciação Formal}

Esta fase foi realizada por uma profissional bilíngue com formação na área de saúde e conhecedora da cultura brasileira e norte-americana. Primeiro, avaliou a equivalência semântica entre o questionário original e cada uma das retraduções, sob a perspectiva do significado referencial das palavras constituintes, para, em seguida, apreciar o significado geral.

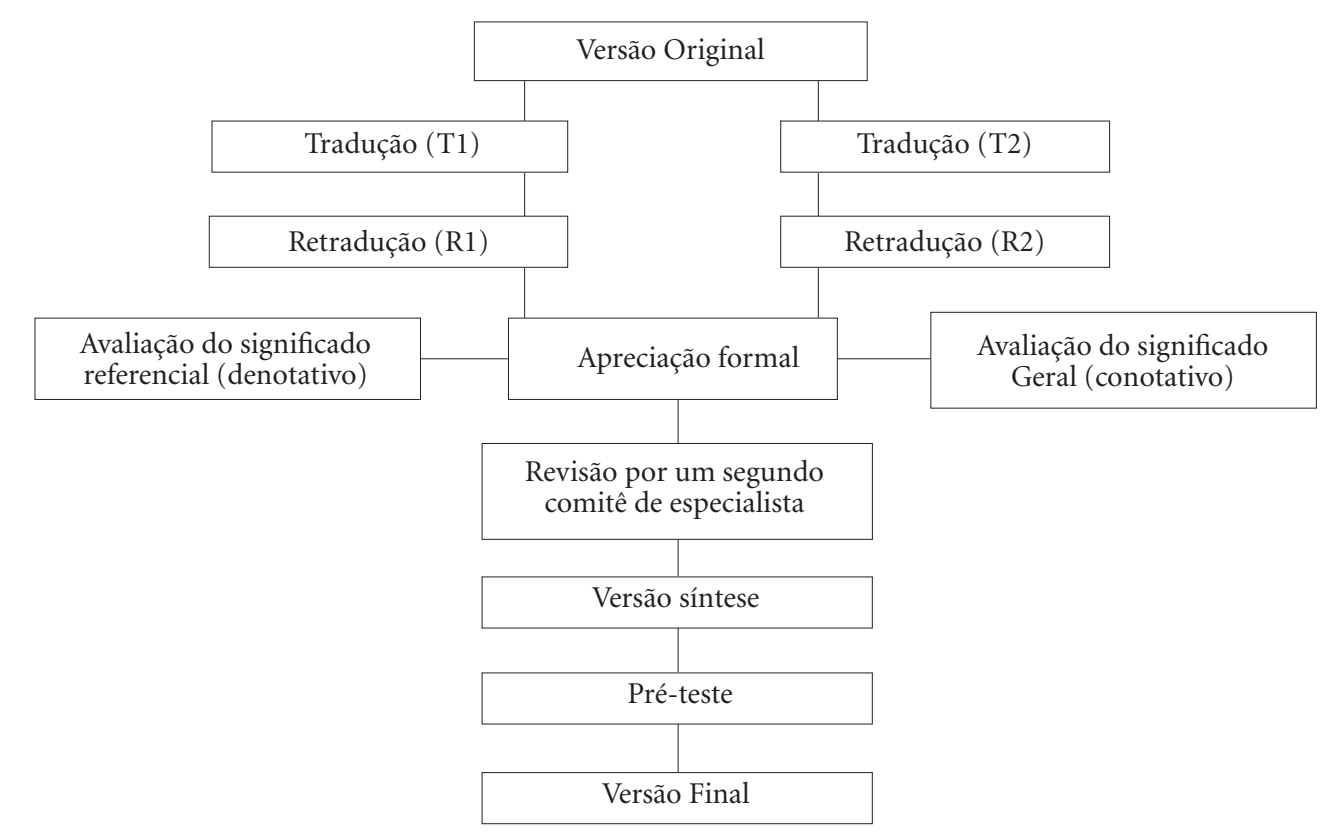

Figura 1. Etapas da equivalência semântica do Observable Indicators of Nursing Home Care Quality Instrument para o português, Rio Grande do Norte, Brasil, 2014. 
No formulário usado para apreciar o significado referencial, a versão original foi comparada com as retraduzidas (inglês - inglês) com o objetivo de avaliar a correspondência literal (denotativa) entre elas. A equivalência entre pares de assertivas foi julgada de forma contínua em uma escala de $0 \%$ a $100 \%$ por ordem crescente de equivalência literal.

O segundo aspecto apreciado representou o significado geral (conotativo) de cada item do IOQ, captado na versão em português em comparação ao original. Essa correspondência transcende a literalidade das palavras, reportando também aspectos mais sutis, como, por exemplo, as significações que determinados termos podem apresentar no contexto cultural da população -alvo. Nesse estudo, optou-se por uma categorização em quatro níveis: inalterado (IN), pouco alterado (PA), muito alterado (MA) ou completamente alterado (CA).

\section{Revisão por um segundo comitê de especialistas}

Nesta fase foi constituído um grupo de quatro profissionais diretamente relacionados ao estudocom o objetivo foi realizar uma análise crítica dos processos anteriores: três de saúde (um doutor especialista na gestão de qualidade em serviços de saúde e dois fisioterapeutas mestrandos no programa de pós-graduação em saúde coletiva) e uma tradutora profissional (licenciada em letras), participante da tradução inicial (T1) do instrumento. Foi verificada a equivalência idiomáticaentre as expressões coloquiais, próprias do idioma, e as operacionais, através da manutenção das características do instrumento original quanto ao número de itens, enunciado e opções de resposta. O produto desta fase foi a versão síntese do instrumento.

\section{Pré-teste}

Executou-se o pré-teste relativo à última fase da equivalência semântica através da aplicação da versão síntese por uma equipe composta por três pares de participantes, representativos de diferentes entes sociais ligados às ILPI: potenciais consumidores, profissionais de saúde e reguladores da Subcoordenadoria de Vigilância Sanitária (SUVISA) do Rio Grande do Norte. O objetivo foi analisar a compreensão dos termos utilizados para então efetuar possíveis ajustes. O IOQ, com o respectivo guia do usuário,foi encaminhado via correio eletrônico. Antes, passou por todas as etapas de adaptação transcultural realizadas neste estudo, exceto pela apreciação formal da equivalência semântica e pelas orientações para padronização das observações. No dia da coleta, foi feita uma leitura seguida de discussão em conjunto para dirimir dúvidas. A amostra foi escolhida por conveniência, sendo constituída por 3 ILPI da cidade de Natal/RN.

\section{Aspectos éticos}

O estudo foi aprovado pelo Comitê de Ética do Hospital Universitário Onofre Lopes (CEP/ HUOL) da Universidade Federal do Rio Grande do Norte (UFRN).

\section{Resultados}

\section{Equivalência conceitual e de itens}

Os itens e o questionário em geral foram considerados pertinentes perante os avaliadores que compuseram o comitê de especialista utilizando o IVC, uma vez que todos os IVC-I superaram o padrão mínimo de aprovação de $80 \%$, bem como o IVC-Q, que ultrapassou $90 \%$. Todavia, alguns itens inicialmente reprovados foram apreciados a partir da adaptação da Técnica de Grupo Nominal, sendo aprovados em uma segunda etapa de avaliação (resultados detalhados estão descritos na Tabela 1).

Alguns itens não obtiveram consenso imediato sobre a validade; por exemplo, no Item 7 , "Você viu enfermeiros registrados?", o grupo aprovou que fosse modificado para "Há enfermeiros na instituição?". O motivo é que o conselho regional de enfermagem exige que todos os enfermeiros sejam registrados e que poderia haver enfermeiros que não tivessem à vista na hora da coleta de dados. No Item 8, "Os enfermeiros registrados parecem conhecer os moradores para que eles sejam capazes de dirigir os seus cuidados?", foi substituído por "Os cuidadores parecem conhecer os moradores para que eles sejam capazes de dirigir os seus cuidados", justificado pelo contexto local, em que a assistência ao idoso está vinculada aos cuidadores e muitas ILPI sequer possuem enfermeiros na equipe de trabalho. Os Itens 27 "Havia animais de estimação (cães, gatos, pássaros, etc.) e/ou plantas naturais na instituição?" e 28 "Os animais e/ou plantas naturais estavam em boas condições?", foram aprovados somente após discussão baseada em estudos científicos ${ }^{10,37}$ e na Lei No 5123referente ao código sanitário ${ }^{38}$. 
Tabela 1. Índice de Validade do Conteúdo da equivalência conceitual e de Itens referente aos critérios de relevância e viabilidade do IOQ total e a cada item, Rio Grande do Norte, Brasil, 2014.

\begin{tabular}{|c|c|c|c|c|c|c|}
\hline \multirow{2}{*}{$\begin{array}{c}\text { Itens do } \\
\text { IOQ }\end{array}$} & \multicolumn{3}{|c|}{$\begin{array}{l}\text { Equivalência conceitual e de itens } \\
\text { (viabilidade) }\end{array}$} & \multicolumn{3}{|c|}{$\begin{array}{l}\text { Equivalência conceitual e de itens } \\
\text { (relevância) }\end{array}$} \\
\hline & $\begin{array}{l}\text { No reprovações } \\
\text { (avaliação 1) }\end{array}$ & $\begin{array}{l}\text { No reprovações } \\
(\text { avaliação 2) }\end{array}$ & $\begin{array}{l}\text { IVC-I } \\
(\%)\end{array}$ & $\begin{array}{l}\text { No reprovações } \\
\text { (avaliação 1) }\end{array}$ & $\begin{array}{l}\text { No reprovações } \\
\text { (avaliação 2) }\end{array}$ & $\begin{array}{c}\text { IVC-I } \\
(\%)\end{array}$ \\
\hline 1 & 0 & - & 100 & 1 & - & 90 \\
\hline 2 & 0 & - & 100 & 0 & - & 100 \\
\hline 3 & 0 & - & 100 & 0 & - & 100 \\
\hline 4 & 1 & - & 90 & 1 & - & 90 \\
\hline 5 & 0 & - & 100 & 0 & - & 100 \\
\hline 6 & 1 & - & 90 & 1 & - & 90 \\
\hline 7 & 5 & 0 & 100 & 3 & 0 & 100 \\
\hline 8 & 4 & 0 & 100 & 2 & - & 80 \\
\hline 9 & 0 & - & 90 & 1 & - & 90 \\
\hline 10 & 0 & - & 100 & 0 & - & 100 \\
\hline 11 & 2 & - & 100 & 0 & - & 100 \\
\hline 12 & 0 & - & 80 & 1 & - & 90 \\
\hline 13 & 0 & - & 100 & 0 & - & 100 \\
\hline 14 & 0 & - & 100 & 0 & - & 100 \\
\hline 15 & 1 & - & 90 & 0 & - & 100 \\
\hline 16 & 0 & - & 100 & 1 & - & 90 \\
\hline 17 & 0 & - & 100 & 0 & - & 100 \\
\hline 18 & 1 & - & 90 & 0 & - & 100 \\
\hline 19 & 2 & - & 80 & 0 & - & 100 \\
\hline 20 & 1 & - & 90 & 0 & - & 100 \\
\hline 21 & 0 & - & 100 & 0 & - & 100 \\
\hline 22 & 2 & - & 80 & 0 & - & 100 \\
\hline 23 & 0 & - & 100 & 1 & - & 90 \\
\hline 24 & 1 & - & 90 & 1 & - & 90 \\
\hline 25 & 1 & - & 90 & 1 & - & 90 \\
\hline 26 & 1 & - & 90 & 0 & - & 100 \\
\hline 27 & 5 & 0 & 100 & 3 & 0 & 100 \\
\hline 28 & 5 & 0 & 100 & 4 & 0 & 100 \\
\hline 29 & 2 & - & 80 & 2 & - & 80 \\
\hline 30 & 1 & - & 90 & 1 & - & 90 \\
\hline IVC-Q (\%) & \multicolumn{3}{|c|}{94,33} & \multicolumn{3}{|c|}{95,33} \\
\hline
\end{tabular}

IVC-I (Índice de validade de conteúdo por item); IVC-Q (Índice de validade de conteúdo do questionário completo).

\section{Equivalência semântica, idiomática e operacional}

Após a realização das traduções e retraduções, realizou-se uma apreciação formal da equivalência referente aos significados geral e referencial. No geral, evidenciou-se uma boa equivalência como ilustra a Tabela 2. Quanto ao significado referencial, observou-se similaridade entre as duas retraduções e o instrumento original, variando entre 90-100\% para a retradução 1 (R1) e 70-100\% para a retradução 2 (R2). Considerando o significado geral, a tradução 1 (T1) foi melhor avaliada, recebendo classificação “inalterado" em $80 \%$ dos itens, enquanto a tradução 2 (T2) apresentou apenas $47 \%$. Porém, nenhum dos itens foi considerado muito alterado.

Ainda em relação à etapa da apreciação formal, a profissional responsável realizou algumas observações comuns. Grande parte da versão 2 ( T2 + R2) estava no presente do indicativo, e não no pretérito imperfeito como no questionário original, além de uma ausência de padronização nas traduções do termo staff que fora representa- 
Tabela 2. Avaliação da equivalência semântica através da apreciação da equivalência referencial e geral entre os itens das retraduções e o instrumento IOQ original, Rio Grande do Norte, Brasil, 2014.

\begin{tabular}{|c|c|c|c|}
\hline & $\begin{array}{l}\text { Julgamento do } \\
\text { avaliador }\end{array}$ & $\begin{array}{c}\text { Versão } 1(\mathrm{~T} 1+\mathrm{R} 1) \\
\text { Número de itens }(\%)\end{array}$ & $\begin{array}{c}\text { Versão } 2 \text { (T2 +R2) } \\
\text { Número de itens (\%) }\end{array}$ \\
\hline \multirow{5}{*}{$\begin{array}{l}\text { Significado Referencial } \\
\text { (original/ retradução) }\end{array}$} & $90-100 \%$ & $30(100)$ & $22(73)$ \\
\hline & $70<90 \%$ & - & $08(27)$ \\
\hline & $50<70 \%$ & - & - \\
\hline & $<50 \%$ & - & - \\
\hline & Total & $30(100)$ & $30(100)$ \\
\hline \multirow{5}{*}{$\begin{array}{l}\text { Significado Geral } \\
\text { (original/ tradução) }\end{array}$} & Inalterado & $24(80)$ & $14(47)$ \\
\hline & Pouco alterado & $06(20)$ & $16(53)$ \\
\hline & Muito alterado & - & - \\
\hline & Completamente alterado & - & - \\
\hline & Total & $30(100)$ & $30(100)$ \\
\hline
\end{tabular}

T1/R1- Itens da Tradução 1 e Retradução 1; T2/R2 - itens da Tradução 2 e Retradução 2.

do como equipe, pessoal ou funcionários. Também foram observadas contrações na R1 que deixariam o texto menos formal. No geral, primou-se pela T1 (67\% dos casos) para estabelecer uma versão síntese após a apreciação formal. Em outras 30\% das situações, a versão T1 e T2 eram correspondentes e em apenas um item optou-se pela versão 2 .

As modificações realizadas tiveram diferentes objetivos, porém, de maneira geral, buscou-se tornar o questionário mais claro e compreensivo parao público alvo. A fim de padronizar o IOQ determinou-se a tradução do termo staff para "equipe de trabalho", bem como o termo residents para "residentes" apenas. O tempo verbal da versão síntese foi o pretérito imperfeito e não o presente do indicativo como em algumas traduções da versão 2. Ademais, foi dado preferência a frases na voz ativa para contemplar a ordem natural dos fatos, facilitando assim o entendimento do IOQ por diferentes entes sociais.

Outras alterações foram mais pontuais. Por exemplo, no item 5 foi preferida a palavra "amável" a "cuidadoso" por expressar de forma mais direta que o residente não necessita apenas de cuidado, mas também de uma maior atenção, inclusive afetuosa. No Item 8 , foi preterido o termo "direcionar" por "gerenciar", pois este remete a uma função mais autêntica do enfermeiro que é de coordenar o cuidado, e não apenas orientá -lo. Neste item foi reconsiderado a mudança do termo "enfermeiros" por "cuidadores" durante a revisão por um segundo comitê de especialistas, apesar da validade de conteúdo dada pelo comitê de especialista 1 na equivalência conceitual e de itens. Essa mudança foi justificada pela existência do enfermeiro na ILPI como um fator diretamente relacionado à qualidade do cuidado, pois a este profissional é atribuída a função de gerenciar o cuidado e não aos cuidadores. No Item 22, optou-se pelo termo "área interna da instituição" em vez de "dentro de casa", em concordância com a RDC 283/2005(Anvisa, 2005) e por deixar mais claro que a referência do local é dentro da área coberta da ILPI. Igualmente, esta foi a escolha referente ao termo "área externa da instituição".

Nas opções de respostas do IOQ também foram realizadas alterações pontuais, como em relação ao termo rarely seen, presente em 6 itens, que foi traduzido de formas diferentes a depender do contexto. Na opção de resposta 1 do Item 7 "Foram vistos enfermeiros nas áreas comuns da instituição? (Observe os crachás ou pergunte à equipe de trabalho)" considerou-se como "não", pois para esse indicador, mesmo em uma breve visita, pode-se afirmar a inexistência permanente de enfermeiros na instituição perguntando à equipe de trabalho, diferente da tradução utilizada "raramente vistos" às demais opções de respostas dos Itens $(9,10,11,12$ e 30), pois nesses casos mesmo não observando os elementos dos itens durante a aplicação do IOQ, podem em outras circunstâncias estarem presentes.O termo "desobstruídos", encontrados nas opções de respostas do Item 17, foi preterido à palavra "desorganizados", por esta ser mais comumente empregada para relatar ambientes livres e sem obstáculos à locomoção. 
O pré-teste foi realizado por três pares de avaliadores em três ILPI no município de Natal/ RN com o objetivo de avaliar a compreensão e a clareza do questionário. Apesar de não haver limitações claras na aplicação e compreensão do IOQ, foi relatada dificuldades no preenchimento da dimensão referente à comunicação interpessoal por necessitar de uma observação mais minuciosa. Observou-se, após o término dessa fase, algumas questões em branco referentes aos pares dos potenciais consumidores, talvez devido à apresentação do IOQ conforme instrumento original em oito páginas. Diante disso, destacou-se a necessidade de deixar o preenchimento da dimensão alusiva à comunicação interpessoal por último, pois esta necessita de um tempo maior para observação. Ademais, alguns ajustes foram feitos a fim de tornar o instrumento mais direto e menos extenso (resultados detalhados estão descritos no Quadro 1).

\section{Discussão}

Os resultados deste estudo são uma contribuição para a melhoria da qualidade dos serviços nas ILPI no contexto brasileiro. Com base nas pesquisas metodológicas de validação de instrumento que foram indicados na revisão da literatura e nas discussões técnicas, atingiu-se satisfatoriamente as etapas iniciais e essenciais da adaptação transcultural do IOQ para a língua portuguesa. Embora longo, o procedimento sistemático foi necessário para garantir a produção de uma versão com equivalência cultural referente às etapas propostas no estudo.

O instrumento a ser adaptado transculturalmente limita-se a avaliar a qualidade do cuidado nas ILPI a partir de dados de estrutura e processo, alusivos à dimensão da atenção centrada na pessoa. Mesmo não havendo uma avaliação das demais dimensões da qualidade em saúde: efetividade, eficiência, segurança oportunidade e acesso, estudos evidenciam relação positiva da atenção centrada na pessoa com os clássicos resultados de saúde ${ }^{20}$. Ademais, segundo Donabe$\operatorname{dian}^{39}$, os indicadores de processo nos quais se baseia o IOQ são a única forma direta de mensuração da qualidade do cuidado, pois as pesquisas consideram a tríade estrutura, processo e resultados interdependentes, na qual uma boa estrutura facilita bons processos que promovem bons resultados de saúde ${ }^{40}$.
Outra questão bastante discutida foi quanto à falta de consenso na literatura em relação às etapas da equivalência semântica, uma vez que algumas pesquisas, mesmo se baseando em estudos similares, realizaram a adaptação através de uma síntese das versões traduzidas e retraduzidas, enquanto que outros efetivaram duas traduções e retraduções sem haver uma síntese entre elas. Decidimos dividir a primeira fase da equivalência semântica em duas traduções e retraduções respectivas, por evidenciar discrepâncias relativas durante o processo e para facilitar futuras adaptações ao contexto local.

Apesar do IOQ não avaliar diretamente a satisfação do usuário, o modelo conceitual do instrumento foi desenvolvido a partir das discussões em grupos focais formados por 8-12 participantes compostos por 80 familiares e 16 residentes das ILPI ${ }^{10}$. Acrescentou-se, ainda, aspectos relevantes segundo opiniões dos prestadores de serviços, estando entre estes: administradores, diretores, médicos, consultores, reguladores, promotores de justiça, assistentes sociais e outros profissionais com extensa experiência nas instituições e análise crítica dos pesquisadores envolvidos. Estabelecendo-se um modelo multidimensional capaz de abranger a qualidade do cuidado nas ILPI a partir da dimensão da atenção centrada na pessoa ${ }^{10}$, sendo esta reconhecida internacionalmente e motivo das principais preocupações dos usuários, familiares e responsáveis em detrimento de aspectos de funcionalmente legal e estrutural da instituição.

Um dos estudos mais abrangentes, referente à atenção centrada na pessoa, foi o programa $P i$ cker/Commonwealth, no qual,a partir de uma entrevista de 6000 mil usuários,foram criadas sete dimensões da qualidade ${ }^{41}$. Porém, esse programa foi direcionado a cuidados agudos e a instituições de longa permanência associadas a hospitais. No âmbito das ILPI, embora explorem a atenção centrada na pessoa, em sua maioria, os indicadores para avaliação e monitoramento das instituições refletem uma perspectiva tecnicista e não as percepções dos próprios usuários, como é o caso do IOQ. Essa visão restringe o envolvimento dos idosos nos serviços prestados e o alcance dos resultados na atenção centrada na pessoa. Não obstante, muitos estudos, quando não enfatizam os dados da estrutura para avaliação da qualidade, ressaltam apenas um dos elementos da tríade estrutura, processo e resultados, o que acaba limitando uma avaliação mais efetiva ${ }^{42}$. 
Quadro 1. Versão final do Observable Indicators of Nursing Home Care Quality Instrument (Português), Rio Grande do Norte, Brasil, 2014.

\begin{tabular}{|c|c|c|c|c|c|c|}
\hline \multicolumn{7}{|c|}{ Comunicação interpessoal (I1 a I6) } \\
\hline I1 & $\begin{array}{l}\text { As conversas entre os residentes e a equipe de } \\
\text { trabalho eram amigáveis? }\end{array}$ & $\begin{array}{c}1 \\
\text { A maioria } \\
\text { não era }\end{array}$ & $\begin{array}{c}2 \\
\text { Poucas eram }\end{array}$ & $\begin{array}{l}3 \\
\text { Algumas } \\
\text { eram }\end{array}$ & $\begin{array}{c}4 \\
\text { Muitas eram }\end{array}$ & $\begin{array}{l}5 \\
\text { A maioria } \\
\text { era }\end{array}$ \\
\hline $\mathrm{I} 2$ & $\begin{array}{l}\text { Quando a equipe de trabalho se dirigia aos } \\
\text { residentes, chamava-os pelo nome? }\end{array}$ & $\begin{array}{c}1 \\
\text { A maioria } \\
\text { não }\end{array}$ & $\begin{array}{c}2 \\
\text { Poucos sim }\end{array}$ & $\begin{array}{l}3 \\
\text { Alguns sim }\end{array}$ & $\begin{array}{c}4 \\
\text { Muitos sim }\end{array}$ & $\begin{array}{l}5 \\
\operatorname{sim}\end{array}$ \\
\hline I3 & $\begin{array}{l}\text { Os residentes e a equipe de trabalho conheciam } \\
\text { uns aos outros e pareciam confortáveis entre } \\
\text { si (por exemplo, sorriso, contato visual, toque, } \\
\text { etc.)? }\end{array}$ & $\begin{array}{c}1 \\
\text { A maioria } \\
\text { não }\end{array}$ & $\begin{array}{c}2 \\
\text { Poucos sim }\end{array}$ & $\begin{array}{c}3 \\
\text { Alguns sim }\end{array}$ & $\begin{array}{c}4 \\
\text { Muitos sim }\end{array}$ & $\begin{array}{l}5 \\
\text { A maioria }\end{array}$ \\
\hline I4 & $\begin{array}{l}\text { Os residentes e a equipe de trabalho interagiam } \\
\text { uns com os outros de forma positiva (por } \\
\text { exemplo, conversas, humor, toque, contato } \\
\text { visual, etc.)? }\end{array}$ & $\begin{array}{c}1 \\
\text { A maioria } \\
\text { não }\end{array}$ & $\begin{array}{c}2 \\
\text { Poucos sim }\end{array}$ & $\begin{array}{c}3 \\
\text { Alguns sim }\end{array}$ & $\begin{array}{c}4 \\
\text { Muitos sim }\end{array}$ & $\begin{array}{l}5 \\
\underset{\operatorname{sim}}{\text { A maioria }}\end{array}$ \\
\hline I5 & $\begin{array}{l}\text { A equipe de trabalho parecia ser amável } \\
\text { (compassiva, calorosa, gentil)? }\end{array}$ & $\underset{\text { não }}{\text { A maioria }}$ & $\begin{array}{c}2 \\
\text { Poucos sim }\end{array}$ & $\begin{array}{c}3 \\
\text { Alguns sim }\end{array}$ & $\begin{array}{c}4 \\
\text { Muitos sim }\end{array}$ & $\begin{array}{l}5 \\
\operatorname{sim}\end{array}$ \\
\hline I6 & $\begin{array}{l}\text { A equipe de trabalho tratava os residentes com } \\
\text { dignidade e respeito? }\end{array}$ & $\begin{array}{c}1 \\
\text { A maioria } \\
\text { não }\end{array}$ & $\begin{array}{c}2 \\
\text { Poucos sim }\end{array}$ & $\begin{array}{c}3 \\
\text { Alguns sim }\end{array}$ & $\begin{array}{c}4 \\
\text { Muitos sim }\end{array}$ & $\begin{array}{l}5 \\
\operatorname{sim}\end{array}$ \\
\hline \multicolumn{7}{|c|}{ Total (Some os números circulados e escreva na caixa ao lado) } \\
\hline \multicolumn{7}{|c|}{ Prestação de cuidados (I7 a I12) } \\
\hline I7 & $\begin{array}{l}\text { Foram vistos enfermeiros nas áreas comuns da } \\
\text { instituição? (Observe os crachás ou pergunte à } \\
\text { equipe de trabalho). }\end{array}$ & $\begin{array}{c}1 \\
\text { Não }\end{array}$ & $\begin{array}{l}2 \\
\text { Ocasional- } \\
\text { mente }\end{array}$ & $\begin{array}{l}3 \\
\text { Algumas } \\
\text { vezes }\end{array}$ & $\begin{array}{l}\quad 4 \\
\text { Com } \\
\text { frequência }\end{array}$ & $\begin{array}{l}5 \\
\text { Com muita } \\
\text { frequência }\end{array}$ \\
\hline I8 & $\begin{array}{l}\mathrm{O}(\mathrm{s}) \text { enfermeiro(s) pareciam conhecer os } \\
\text { residentes para gerenciar os seus cuidados? } \\
\text { (Pode ser necessário perguntar à equipe de } \\
\text { trabalho). }\end{array}$ & $\begin{array}{c}1 \\
\text { Não } \\
\text { pareciam }\end{array}$ & $\begin{array}{l}2 \\
\text { Ocasional- } \\
\text { mente }\end{array}$ & $\begin{array}{l}3 \\
\text { Algumas } \\
\text { vezes }\end{array}$ & $\begin{array}{l}\quad 4 \\
\text { Com } \\
\text { frequência }\end{array}$ & $\begin{array}{l}5 \\
\text { Com muita } \\
\text { frequência }\end{array}$ \\
\hline I9 & $\begin{array}{l}\text { A equipe de trabalho ajudava os residentes } \\
\text { com a alimentação? }\end{array}$ & $\begin{array}{c}1 \\
\text { Raramente } \\
\text { visto }\end{array}$ & $\begin{array}{l}2 \\
\text { Ocasional- } \\
\text { mente }\end{array}$ & $\begin{array}{l}3 \\
\text { Algumas } \\
\text { vezes }\end{array}$ & $\begin{array}{l}\quad 4 \\
\text { Com } \\
\text { frequência }\end{array}$ & $\begin{array}{l}5 \\
\text { Com muita } \\
\text { frequência }\end{array}$ \\
\hline $\mathrm{I} 10$ & $\begin{array}{l}\text { Os residentes se deslocavam pela instituição de } \\
\text { forma independente, com ou sem dispositivo } \\
\text { de auxílio, tais como bengalas, andadores ou } \\
\text { cadeiras de rodas? }\end{array}$ & $\begin{array}{c}1 \\
\text { Raramente } \\
\text { visto }\end{array}$ & $\begin{array}{l}2 \\
\text { Ocasional- } \\
\text { mente }\end{array}$ & $\begin{array}{l}3 \\
\text { Algumas } \\
\text { vezes }\end{array}$ & $\begin{array}{l}\quad 4 \\
\text { Com } \\
\text { frequência }\end{array}$ & $\begin{array}{l}5 \\
\text { Com muita } \\
\text { frequência }\end{array}$ \\
\hline I11 & $\begin{array}{l}\text { A equipe de trabalho ajudava alguns residentes } \\
\text { a caminhar ou se locomover pela instituição? }\end{array}$ & $\begin{array}{c}1 \\
\text { Raramente } \\
\text { visto }\end{array}$ & $\begin{array}{l}2 \\
\text { Ocasional- } \\
\text { mente }\end{array}$ & $\begin{array}{l}3 \\
\text { Algumas } \\
\text { vezes }\end{array}$ & $\begin{array}{l}\quad 4 \\
\text { Com } \\
\text { frequência }\end{array}$ & $\begin{array}{l}5 \\
\text { Com muita } \\
\text { frequência }\end{array}$ \\
\hline I12 & $\begin{array}{l}\text { A equipe de trabalho se comunicava com os } \\
\text { residentes confusos de forma positiva (por } \\
\text { exemplo, falar, tocar, sentar-se com eles, etc.)? }\end{array}$ & $\begin{array}{c}1 \\
\text { Raramente } \\
\text { visto }\end{array}$ & $\begin{array}{c}2 \\
\text { Ocasional- } \\
\text { mente }\end{array}$ & $\begin{array}{c}3 \\
\text { Algumas } \\
\text { vezes }\end{array}$ & $\begin{array}{c}4 \\
\text { Com } \\
\text { frequência }\end{array}$ & $\begin{array}{c}5 \\
\text { Com muita } \\
\text { frequência }\end{array}$ \\
\hline
\end{tabular}


Quadro 1. continuação

\begin{tabular}{|c|c|c|c|c|c|c|}
\hline \multicolumn{7}{|c|}{ Gestão da aparência dos idosos (I13 e I14) } \\
\hline $\mathrm{I} 13$ & Os residentes estavam vestidos e limpos? & $\begin{array}{c}1 \\
\text { A maioria } \\
\text { não }\end{array}$ & $\begin{array}{c}2 \\
\text { Alguns } \\
\text { estavam }\end{array}$ & $\begin{array}{c}3 \\
\text { Muitos } \\
\text { estavam }\end{array}$ & $\begin{array}{l}4 \\
\text { A maioria } \\
\text { estava }\end{array}$ & $\begin{array}{c}5 \\
\text { Todos } \\
\text { estavam }\end{array}$ \\
\hline $\mathrm{I} 14$ & $\begin{array}{l}\text { Os residentes aparentavam estar bem cuidados } \\
\text { (barba feita, cabelo penteados, unhas limpas e } \\
\text { aparadas)? }\end{array}$ & $\begin{array}{l}1 \\
\text { A maioria }\end{array}$ & $\begin{array}{l}2 \\
\text { Alguns } \\
\text { estavam }\end{array}$ & $\begin{array}{c}3 \\
\text { Muitos } \\
\text { estavam }\end{array}$ & $\begin{array}{l}4 \\
\text { A maioria } \\
\text { estava }\end{array}$ & $\begin{array}{c}5 \\
\text { Todos } \\
\text { estavam }\end{array}$ \\
\hline \multicolumn{7}{|c|}{ Total (Some os números circulados e escreva na caixa ao lado) } \\
\hline \multicolumn{7}{|c|}{ Odores (I15 e I16) } \\
\hline I15 & $\begin{array}{l}\text { Odores de urina ou fezes eram perceptíveis na } \\
\text { instituição? }\end{array}$ & $\begin{array}{c}1 \\
\text { Domi- } \\
\text { nante em } \\
\text { todas as } \\
\text { áreas }\end{array}$ & $\begin{array}{l}2 \\
\text { Na maioria } \\
\text { das áreas }\end{array}$ & $\begin{array}{c}3 \\
\text { Ocasional- } \\
\text { mente }\end{array}$ & $\begin{array}{c}4 \\
\text { Quase } \\
\text { imperceptível }\end{array}$ & $\begin{array}{l}5 \\
\text { Imperceptível }\end{array}$ \\
\hline I16 & $\begin{array}{l}\text { Outros odores desagradáveis eram perceptíveis } \\
\text { na instituição? }\end{array}$ & $\begin{array}{c}1 \\
\text { Domi- } \\
\text { nante em } \\
\text { todas as } \\
\text { áreas }\end{array}$ & $\begin{array}{c}2 \\
\text { Na maioria } \\
\text { das áreas }\end{array}$ & $\begin{array}{c}3 \\
\text { Ocasional- } \\
\text { mente }\end{array}$ & $\begin{array}{c}4 \\
\text { Quase } \\
\text { imperceptível }\end{array}$ & $\begin{array}{c}5 \\
\text { Imperceptível }\end{array}$ \\
\hline \multicolumn{7}{|c|}{ Total (Some os números circulados e escreva na caixa ao lado) } \\
\hline \multicolumn{7}{|c|}{ Utilização dos ambientes básicos (Q17 a Q21) } \\
\hline I17 & $\begin{array}{l}\text { Os corredores e áreas comuns estavam sem } \\
\text { obstáculos à locomoção? }\end{array}$ & $\begin{array}{c}1 \\
\text { Muito } \\
\text { desorga- } \\
\text { nizados }\end{array}$ & $\begin{array}{c}2 \\
\text { Frequente- } \\
\text { mente } \\
\text { desorga- } \\
\text { nizados }\end{array}$ & $\begin{array}{c}3 \\
\text { Um pouco } \\
\text { desorgani- } \\
\text { zados }\end{array}$ & $\begin{array}{c}4 \\
\text { Organizados }\end{array}$ & $\begin{array}{c}5 \\
\text { Muito } \\
\text { organizados }\end{array}$ \\
\hline I18 & $\begin{array}{l}\text { Os quartos dos residentes, corredores e áreas } \\
\text { comuns estavam limpos? }\end{array}$ & $\begin{array}{c}1 \\
\text { Sujos }\end{array}$ & $\begin{array}{c}2 \\
\text { Um pouco } \\
\text { sujos }\end{array}$ & $\begin{array}{c}3 \\
\text { Um pouco } \\
\text { sujos }\end{array}$ & $\begin{array}{c}4 \\
\text { Limpos }\end{array}$ & $\begin{array}{l}5 \\
\text { Muito limpos }\end{array}$ \\
\hline I19 & $\begin{array}{l}\text { O prédio, o terreno e os móveis da instituição } \\
\text { estavam em bom estado? }\end{array}$ & $\begin{array}{c}1 \\
\text { Condições } \\
\text { muito } \\
\text { ruins }\end{array}$ & $\begin{array}{c}2 \\
\text { Condições } \\
\text { ruins }\end{array}$ & $\begin{array}{c}3 \\
\text { Mais ou } \\
\text { menos } \\
\text { limpos }\end{array}$ & $\begin{array}{c}4 \\
\text { Condições } \\
\text { boas }\end{array}$ & $\begin{array}{c}5 \\
\text { Condições } \\
\text { muito boas }\end{array}$ \\
\hline $\mathrm{I} 20$ & Os corredores estavam bem iluminados? & $\begin{array}{c}1 \\
\text { Mal ilumi- } \\
\text { nados }\end{array}$ & $\begin{array}{c}2 \\
\text { Alguma luz, } \\
\text { mas não o } \\
\text { suficiente }\end{array}$ & $\begin{array}{c}3 \\
\text { Condições } \\
\text { pouco boas }\end{array}$ & $\begin{array}{c}4 \\
\text { Bem } \\
\text { iluminados }\end{array}$ & $\begin{array}{l}\qquad 5 \\
\text { Muito bem } \\
\text { iluminados }\end{array}$ \\
\hline $\mathrm{I} 21$ & $\begin{array}{l}\text { Os quartos dos residentes estavam bem } \\
\text { iluminados? }\end{array}$ & $\begin{array}{c}1 \\
\text { Mal ilumi- } \\
\text { nados }\end{array}$ & $\begin{array}{c}2 \\
\text { Alguma luz, } \\
\text { mas não o } \\
\text { suficiente }\end{array}$ & $\begin{array}{c}3 \\
\text { Modera- } \\
\text { damente } \\
\text { iluminados }\end{array}$ & $\begin{array}{c}4 \\
\text { Bem } \\
\text { iluminados }\end{array}$ & \begin{tabular}{c}
\multicolumn{1}{c}{5} \\
Muito bem \\
iluminados
\end{tabular} \\
\hline
\end{tabular}


Quadro 1. continuação

\begin{tabular}{|c|c|c|c|c|c|c|}
\hline \multicolumn{7}{|c|}{ Acesso aos ambientes das ILPIS (Q22 a Q25) } \\
\hline I 22 & $\begin{array}{l}\text { Os residentes confusos têm um lugar seguro } \\
\text { para passear na área interna da instituição? } \\
\text { (Pode ser necessário perguntar à equipe de } \\
\text { trabalho). }\end{array}$ & $\begin{array}{l}1 \\
\text { Sem lugar } \\
\text { seguro } \\
\text { aparente }\end{array}$ & $\begin{array}{c}2 \\
\text { Muito } \\
\text { pequeno }\end{array}$ & $\begin{array}{c}3 \\
\text { Pequeno }\end{array}$ & $\begin{array}{c}4 \\
\text { Moderado }\end{array}$ & $\begin{array}{l}5 \\
\text { Lugar seguro } \\
\text { e grande }\end{array}$ \\
\hline I 23 & $\begin{array}{l}\text { Os residentes confusos têm um lugar seguro } \\
\text { para passear na área externa da instituição? } \\
\text { (Pode ser necessário perguntas à equipe de } \\
\text { trabalho). }\end{array}$ & $\begin{array}{l}1 \\
\text { Sem lugar } \\
\text { seguro } \\
\text { aparente }\end{array}$ & $\begin{array}{c}2 \\
\text { Muito } \\
\text { pequeno }\end{array}$ & $\begin{array}{c}3 \\
\text { Pequeno }\end{array}$ & $\begin{array}{c}4 \\
\text { Moderado }\end{array}$ & $\begin{array}{l}\quad 5 \\
\text { Lugar seguro } \\
\text { e grande }\end{array}$ \\
\hline I 24 & $\begin{array}{l}\text { Os residentes confusos têm acesso à área } \\
\text { externa da instituição? (Pode ser necessário } \\
\text { perguntar à equipe de trabalho). }\end{array}$ & $\begin{array}{c}1 \\
\text { Sem acesso } \\
\text { aparente }\end{array}$ & $\begin{array}{c}2 \\
\text { Acesso } \\
\text { ocasional } \\
\text { com } \\
\text { assistência }\end{array}$ & $\begin{array}{c}3 \\
\text { Algum } \\
\text { acesso com } \\
\text { assistência }\end{array}$ & $\begin{array}{l}\quad 4 \\
\text { Acesso } \\
\text { frequente }\end{array}$ & $\begin{array}{c}5 \\
\text { Acesso a } \\
\text { qualquer } \\
\text { hora }\end{array}$ \\
\hline I 25 & $\begin{array}{l}\text { Os outros residentes têm acesso à área } \\
\text { externa da instituição? (Pode ser necessário } \\
\text { perguntar à equipe de trabalho). }\end{array}$ & $\begin{array}{c}1 \\
\text { Sem acesso } \\
\text { aparente }\end{array}$ & $\begin{array}{c}2 \\
\text { Acesso } \\
\text { ocasional } \\
\text { com } \\
\text { assistência }\end{array}$ & $\begin{array}{c}3 \\
\text { Algum } \\
\text { acesso com } \\
\text { assistência }\end{array}$ & $\begin{array}{l}\quad 4 \\
\text { Acesso } \\
\text { frequente }\end{array}$ & $\begin{array}{c}5 \\
\text { Acesso a } \\
\text { qualquer } \\
\text { hora }\end{array}$ \\
\hline \multicolumn{7}{|c|}{ Total (Some os números circulados e escreva na caixa ao lado) } \\
\hline \multicolumn{7}{|c|}{ Ambiente familiar (Q26 a Q30) } \\
\hline I 26 & $\begin{array}{l}\text { Os quartos dos residentes estavam } \\
\text { personalizados com mobiliário, imagens e } \\
\text { outros objetos pessoais? }\end{array}$ & $\begin{array}{c}1 \\
\text { A maioria } \\
\text { não estava }\end{array}$ & $\begin{array}{c}2 \\
\text { Poucos } \\
\text { estavam }\end{array}$ & $\begin{array}{c}3 \\
\text { Alguns } \\
\text { estavam }\end{array}$ & $\begin{array}{c}4 \\
\text { Muitos } \\
\text { estavam }\end{array}$ & $\begin{array}{l}5 \\
\text { A maioria } \\
\text { estava }\end{array}$ \\
\hline I 27 & $\begin{array}{l}\text { Havia animais de estimação (cães, gatos, } \\
\text { pássaros, etc.) e/ou plantas naturais na } \\
\text { instituição? }\end{array}$ & $\begin{array}{c}1 \\
\text { Nenhum } \\
\text { ou } \\
\text { raramente } \\
\text { vistos }\end{array}$ & $\begin{array}{l}2 \\
\text { Ocasional- } \\
\text { mente }\end{array}$ & $\begin{array}{l}3 \\
\text { Algumas } \\
\text { vezes }\end{array}$ & $\begin{array}{l}4 \\
\text { Com } \\
\text { frequência }\end{array}$ & $\begin{array}{l}5 \\
\text { Com muita } \\
\text { frequência }\end{array}$ \\
\hline $\mathrm{I} 28$ & $\begin{array}{l}\text { Os animais e/ou plantas naturais estavam em } \\
\text { boas condições? }\end{array}$ & $\begin{array}{c}1 \\
\text { Não vistos } \\
\text { ou em } \\
\text { condições } \\
\text { ruins }\end{array}$ & $\begin{array}{l}2 \\
\text { Condições } \\
\text { razoáveis }\end{array}$ & $\begin{array}{c}3 \\
\text { Mediano }\end{array}$ & $\begin{array}{l}4 \\
\text { Condições } \\
\text { boas }\end{array}$ & $\begin{array}{c}5 \\
\text { Condições } \\
\text { muitos boas }\end{array}$ \\
\hline I 29 & Havia uma aparência familiar na instituição? & $\begin{array}{c}1 \\
\text { Nada } \\
\text { familiar }\end{array}$ & $\begin{array}{c}2 \\
\text { Pouco } \\
\text { familiar }\end{array}$ & $\begin{array}{c}3 \\
\text { Moderada- } \\
\text { mente } \\
\text { familiar }\end{array}$ & $\begin{array}{l}4 \\
\text { Aparência } \\
\text { familiar }\end{array}$ & $\begin{array}{l}5 \\
\text { Muito } \\
\text { familiar }\end{array}$ \\
\hline $\mathrm{I} 30$ & $\begin{array}{l}\text { Visitantes foram vistos na instituição } \\
\text { (familiares, voluntários, membros da } \\
\text { comunidade, etc.)? }\end{array}$ & $\begin{array}{l}1 \\
\text { Raramente } \\
\text { vistos }\end{array}$ & $\begin{array}{l}2 \\
\text { Ocasional- } \\
\text { mente }\end{array}$ & $\begin{array}{l}3 \\
\text { Algumas } \\
\text { vezes }\end{array}$ & $\begin{array}{l}\quad 4 \\
\text { Frequente- } \\
\text { mente }\end{array}$ & $\begin{array}{l}5 \\
\text { Muito } \\
\text { frequente- } \\
\text { mente }\end{array}$ \\
\hline
\end{tabular}


No geral, os resultados desse estudo de adaptação transcultural do Observable Indicators of Nursing Home Care Quality Instrument mostraram-se positivos e de simples entendimento. Os processos de equivalência conceitual e de itens do instrumento apresentaram-se relevante $(95,33 \%)$ e viável $(94,33 \%)$ para o contexto local, uma vez que o IVC-I e o IVC-Q tiveram resultados superiores aos parâmetros de aprovação indicados na literatura $^{36}$. A compreensão e a clareza do IOQ, aferidas durante a aplicação do pré-teste, revelaram que as etapas iniciais para adaptação foram cumpridas com êxito. Apesar da grande extensão referente à etapa da equivalência semântica e idiomática, o cuidado em adaptar as expressões coloquiais do inglês ao contexto brasileiro foi essencial para que pudéssemos adequar o instrumento.

Na mesma linha de argumentação, destaca-se a manutenção das características do IOQ através da semelhança estrutural do layout, modo de aplicação, pontuação de cada item, dimensão e instrumento completo, bem como a adaptação do guia do usuário para orientar melhor os diferentes entes sociais. Assume-se a equivalência operacional como realizada.

De origem norte-americana, o IOQ também é utilizado na Islândia, Canadá ${ }^{43} \mathrm{e}$ foi adaptado à Coreia do Sul ${ }^{44}$. A utilidade do instrumento aplica-se a reguladores, profissionais e consumidores dos serviços das ILPI. No âmbito brasileiro, o IOQ se apresenta como um instrumento original e de suma importância para a nova filosofia da Agência Nacional de Vigilância Sanitária de repensar os processos assistenciais através de uma cultura de monitoramento avaliativo ${ }^{45}$. No con- texto de crescente foco na redução de custos em cuidados de saúde e em agregar valor aos recursos gastos, torna-se imperativo que se possa avaliar a qualidade do cuidado (externa ou internamente) de forma rápida e eficaz, tanto pelos reguladores como pelos profissionais das próprias ILPI. Além disso, o IOQ foi desenvolvido de forma que mesmo os consumidores com pouquíssimo conhecimento sobre as Instituições de Longa Permanência para Idosos possam utilizá-lo para auxiliar as tomadas de decisões para si ou para membros da família, embasados na qualidade dos cuidados das instituições avaliadas.

\section{Considerações finais}

O IOQ é um instrumento que proporciona uma avaliação da qualidade do cuidado nas ILPI segundo os aspectos valorizados pelos seus usuários, seguindo a lógica da qualidade do cuidado centrado na pessoa. Devido à ausência, no Brasil, de instrumentos válidos e confiáveis para avaliar o cuidado neste âmbito, este estudo tem papel inovador e promissor. A adaptação, segundo a perspectiva de diferentes entes sociais (potenciais consumidores, profissionais de saúde e reguladores), oportuniza uma utilização ampla do instrumento. Após cumprida as fases iniciais de adaptação transcultural neste estudo, o processo de validação necessita ser complementado com análise de sua equivalência de mensuração, ou seja, estudos empíricos de suas propriedades psicométricas tais como a confiabilidade e a validade da estrutura dimensional do instrumento.

\section{Colaboradores}

WIF Oliveira e ZAS Gama participaram da redação, concepção, delineamento do estudo e análise dos dados. G Piuvezam, WIF Oliveira e ZAS Gama participaram da revisão do texto e aprovação da versão final. 


\section{Referências}

1. Nogueira SL, Ribeiro RC, Rosado LE, Franceschini SC, Ribeiro AQ, Pereira ET. Determinant factors of functional status among the oldest old. Rev Bras Fisioter 2010; 14(4):322-329.

2. Instituto Brasileiro de Geografia e Estatística (IBGE). Coordenação de População e Indicadores Sociais. Projeção da população do Brasil por sexo e idade para o período 2000/2060: projeção da população das unidades da federação por sexo e idade para o período 2000/2030. Rio de Janeiro: IBGE; 2013.

3. Costa EFA. Análise de Soroprevalência para as infecções pelos vírus das hepatites $\mathrm{B}$ e C em idoso residentes em asilos no município de Goiânia-GO [dissertação]. Goiânia: Universidade de Goiás; 2004.

4. Committee on Improving Quality in Long-term Care. Institute of Medicine (US). Improving the quality of long-term care. Washington: National Academy Press; 2001.

5. Christophe M, Camarano AA. Dos asilos às instituições de longa permanência: uma história de mitos e preconceitos. In: Camarano AA, organizador. Cuidados de longa duração para a população idosa: um novo risco social a ser assumido? Rio de Janeiro: IPEA; 2010. p. 146-162.

6. Araújo CLO, Souza LAS, Faro ACM. Trajetória das instituições de longa permanência para idosos no Brasil. Hist Enferm Rev Eletrônica 2010; 1(2):250-262.

7. United States General Accounting Office. Nursing homes: quality of care more related to staffing than spending. Washington: GAO Office; 2002.

8. Committee on Quality of Health Care in America. Institute of Medicine (U.S.). Crossing the quality chasm: a new health system for the 21st century. Washington: National Academy Press; 2001.

9. Juran JM. Conceptos básicos. In: Juran JM, Gryna FM, Binghan RS, organizadores. Manual de Control de la Calidad. 2a ed. Barcelona: Reverte; 1990. p. 5-26.

10. Rantz MJ, Zwygart-Stauffacher M, Popejoy L, Grando VT, Mehr DR, Hicks LL, Conn VS, Wipke-Tevis D, Porter R, Bostick J, Maas M, Scott J. Nursing home care quality: a multidimensional theoretical model integrating the views of consumers and providers. J Nurs Care Qual 1999; 14(1):16-37.

11. World Health Organization (WHO). The World Health Report 2000: Health Systems: Improving Performance. Geneva: WHO; 2000.

12. Arah OA, Westert GP, Hurst J, Klazinga NS. A conceptual framework for the OECD Health Care Quality Indicators Project. Int J Qual Health Care 2006; 18(Supl. 1):5-13.

13. International Alliance of Patients' Organizations. What is Patient-Centred Health Care? A Review of Definitions and Principles. $2^{\mathrm{a}}$ ed. London: IAPO; 2007.

14. Bauman A, Fardy H, Harris P. Getting it right: why bother with patient-centred care? Med J Aust 2003; 179(5):253-256.

15. United States of America. Institute for Patient- and Family-Centered Care. Advancing the practice of patient- and family-centered care: how to get started. Bethesda: Institute for Patient-and Family-Centered Care; 2008 .

16. Longtin Y, Sax H, Leape LL, Sheridan SE, Donaldson L, Pittet D. Patient Participation: current knowledge and applicability to patient safety. Mayo Clin Proc 2010; 85(1):53-62.
17. Stewart M, Brown J, Donner A, McWhinney I, Oates J, Weston W, Jordan J. The impact of patient-centered care on outcomes. J Fam Pract 2000; 49(9):796-804.

18. Stewart M. Towards a global definition of patient centred care. Br Med J 2001; 322(7284):444-445.

19. Berwick DM. What 'patient-centered' should mean: confessions of an extremist. Health aff 2009; 28(4): w555-651.

20. Epstein RM, Street RL. Patient-Centered Care for the 21st Century: Physicians' Roles, Health Systems and Patients' Preferences. Philadelphia: American Board of Internal Medicine Foundation; 2008.

21. Andersson I, Pettersson E, Sidenvall B. Daily life after moving into a care home--experiences from older people, relatives and contact persons. J Clin Nurs 2007; 16(9):1712-1718.

22. Bergland A, Kirkevold M. Thriving in nursing homes in Norway: contributing aspects described by residents. Int J Nurs Stud 2006; 43(6):681-691.

23. Hjaltadottir I, Gustafsdottir M. Quality of life in nursing homes: perception of physically frail elderly residents. Scand J Caring Sci 2007; 21(1):48-55.

24. Rantz MJ, Zwygart-Stauffacher M, Mehr DR, Petroski GF, Owen SV, Madsen RW, Flesner M, Conn V, Bostick J, Smith R, Maas M. Field testing, refinement, and psychometric evaluation of a new measure of nursing home care quality. J Nurs Meas 2006; 14(2):129-148.

25. Rantz MJ, Zwygart-Stauffacher M. How to Find the Best Eldercare: A 20-Minute Guide to Assisted Living, In-Home Care, Nursing Homes, \& Senior Housing In Your Community. In: Nursing Homes: making a decicion about quality of care. Minneapolis: Fairview Press; 2009. p. 103-172.

26. Brasil. Ministério da Saúde (MS). Agência Nacional de Vigilância Sanitária. Resolução da Diretoria Colegiada - RDC 283. Brasília: MS; 2005.

27. Felician AM, Santos SSC, Pelzer MT, Oliveira AMN, Pinho LB. Construção de ferramenta avaliativa direcionada às instituições de longa permanência para idosos: relato de experiência. Rev Eletr Enf 2011; 13(3):474482.

28. Beaton DE, Bombardier C, Guillemin F, Ferraz MB. Guidelines for the process of cross-cultural adaptation of self-report measures. Spine 2000; 25(24):3186-3191.

29. Herdman M, Fox-Rushby J, Badia X. 'Equivalence' and the translation and adaptation of health-related quality of life questionnaires. Qual Life Res 1997; 6(3):237-247.

30. Herdman M, Fox-Rushby J, Badia X. A model of equivalence in the cultural adaptation of HRQoL instruments: the universalist approach. Qual Life Res 1998; 7(4):323-335.

31. Reichenheim ME, Moraes CL. Operacionalização de adaptação transcultural de instrumentos de aferição usados em epidemiologia. Rev Saude Publica 2007; 41(4):665-673.

32. Hutchinson A, Bentzen N, Konig-Zanhn C. Cross cultural health outcome assessment: a user's guide. Amsterdan: ERGHO; 1996.

33. Lynn MR. Determination and quantification of content validity. Nurs Res 1986; 35(6):382-385.

34. Hartz ZMA, Vieira da Silva LM, organizadoras. Avaliação em saúde: dos modelos teóricos à prática na avaliação de programas e sistemas de saúde. $2^{\text {a }}$ ed. Salvador: EDUFBA; 2008 . 
35. Wynd CA, Schmidt B, Schaefer MA. Two quantitative approaches for estimating content validity. West J Nurs Res 2003; 25(5):508-518.

36. Polit DF, Beck CT. The content validity index: are you sure you know what's being reported? Critique and recommendations. Res Nurs health 2006; 29(5):489-497.

37. Colombo G, Buono MD, Smania K, Raviola R, De Leo D. Pet therapy and institutionalized elderly: a study on 144 cognitively unimpaired subjects. Arch Gerontol Geriatr 2006; 42(2):207-216.

38. Natal. Lei no 5132, de 29 de setembro de 1999. Dispõe sobre as alterações na Lei no 4.041 , de 13 de novembro de 1991, que trata das atribuições do Município do Natal, e aprova as normas de promoção, proteção e recuperação da Saúde e dá outras providências. 1999. Diário Oficial do Município 1999; 30 set.

39. Donabedian A. The quality of care. How can it be assessed? JAMA 1988;260(12):1743-1748.

40. Cowing M, Davino-Ramaya CM, Ramaya K, Szmerekovsky J. Health care delivery performance: service, outcomes, and resource stewardship. Perm J 2009; 13(4):72-78.

41. Gerteis M, Edgman-Levitan S, Daley J, DelbancoTL, editors. Through the patient's eyes. San Francisco: Jossey-Bass; 1993.

42. Wunderlich GS, Kohler PO, editors. Improving the quality of long-term care. Washington: National Academy Press; 2001.

43. Rantz M, Jensdottir AB, Hjaltadottir I, Gudmundsdottir H, Sigurveig Gudjonsdottir J, Brunton B, Rook M. International field test results of the Observable Indicators of Nursing home care quality instrument. Int Nurs Rev 2002; 49(4):234-242.

44. Lee J. Validity and reliability of the Korean version of the observable indicators of nursing home care quality evaluation instrument. Taehan Kanho Hakhoe Chi 2008; 38(3):474-482.

45. Anvisa. Boletim Informativo: segurança do paciente e qualidade dos serviços em saúde. Anvisa 2011; 1(1):112. 